\title{
Fruit and vegetable intake and breast cancer prognosis: a meta-analysis of prospective cohort studies
}

\author{
Chen Peng ${ }^{1}$, Wei-Ping Luo ${ }^{1,2}$ and Cai-Xia Zhang ${ }^{1,3 *}$ \\ ${ }^{1}$ Department of Medical Statistics and Epidemiology, School of Public Health, Sun Yat-Sen University, Guangzhou 510080, \\ People's Republic of China \\ ${ }^{2}$ Department of Prevention and Health Care, Guangzhou First People's Hospital, Guangzhou Medical University, Guangzhou \\ 510180, People's Republic of China \\ ${ }^{3}$ Guangdong Provincial Key Laboratory of Food, Nutrition and Health, Guangzhou 510080, People's Republic of China
}

(Submitted 7 October 2016 - Final revision received 20 January 2017 - Accepted 3 February 2017)

\section{Abstract}

The effect of fruit and vegetable intake on breast cancer prognosis is controversial. Thus, a meta-analysis was carried out to explore their associations. A comprehensive search was conducted in PubMed, Web of Science, OVID, ProQuest and Chinese databases from inception to April 2016. The summary hazard ratios (HR) and $95 \%$ CI were estimated using a random effects model if substantial heterogeneity existed and using a fixed effects model if not. Subgroup analyses and sensitivity analyses were also performed. In total, twelve studies comprising 41185 participants were included in the meta-analysis. Comparing the highest with the lowest, the summary HR for all-cause mortality were $1.01(95 \% \mathrm{CI} 0.72,1.42)$ for fruits and vegetables combined, 0.96 (95\% CI 0.83, 1.12) for total vegetable intake, $0.99(95 \%$ CI 0.89, 1.11) for cruciferous vegetable intake and 0.88 (95\% CI 0.74, 1.05) for fruit intake; those for breast cancer-specific mortality were 1.05 (95\% CI 0.77, 1.43) for total vegetable intake and $0.94(95 \% \mathrm{CI}$ $0.69,1.26)$ for fruit intake; and those for breast cancer recurrence were 0.89 (95\% CI 0.53, 1.50) for total vegetable intake and 0.98 (95\% CI $0.76,1.26$ ) for cruciferous vegetable intake. This meta-analysis found no significant associations between fruit and vegetable intake and breast cancer prognosis.

Key words: Fruits and vegetables: Breast cancer: Recurrence: Mortality: Meta-analyses

Breast cancer is the most prevalent cancer among women; it is increasing globally and is the most common cause of cancerrelated death in women on all continents ${ }^{(1)}$. Breast cancer affects about $12 \%$ of women worldwide ${ }^{(2)}$. It was reported that 1.7 million new cases were diagnosed worldwide in 2012, accounting for about $25 \%$ of total cancer cases in women ${ }^{(3)}$. Moreover, breast cancer is the leading cause of cancer deaths in women. It has the highest mortality of any cancer in women (12.9 per 100000 ), accounting for approximately $14.7 \%$ of all cancer-related mortalities among women worldwide ${ }^{(4)}$. However, advances in early detection and in types of therapies and their application have resulted in prolonged survival among women diagnosed with breast cancer ${ }^{(5)}$. Thus, it is very important to research the prognostic factors of breast cancer given the increasing number of breast cancer survivors.

Fruits and vegetables may offer potential protective effects against breast cancer occurrence and prognosis. Various biochemical compositions found in a diet high in fruits and vegetables kill breast cancer cells in vitro and prevent and suppress breast cancer progression in various preclinical animal models ${ }^{(6)}$. Chatterjee et $a l .{ }^{(7)}$ found that carotenoids, consisting largely of $\alpha$-carotene, $\beta$-carotene and $\beta$-cryptoxanthin, are typical constituents of orange-, red- and yellow-coloured fruits and green vegetables. $\beta$-Ionone, an end-ring analogue of $\beta$-carotenoid, inhibits 7,12-dimethylbenz- $(\alpha)$ anthracene (DMBA)induced mammary carcinogenesis by inhibiting cell proliferation and inducing apoptosis ${ }^{(8)}$. Liu et $a l^{(9)}$ also documented the chemopreventive effects of varied doses of dietary $\beta$-ionone on the development and growth of DMBA-induced rat mammary tumours and the biologically relevant plasma antioxidant status. Cruciferous vegetables are also major sources of glucosinolatederived bioactive compounds such as isothiocyanates, which have been shown in animal and in vitro studies to inhibit cancer growth and progression ${ }^{(10)}$. Sulforaphane is an isothiocyanate that elicits both pro-apoptotic and anti-proliferative properties ${ }^{(11)}$. Azarenko ${ }^{(12)}$ found that sulforaphane stabilised microtubules in breast cancer cells by suppressing microtubule dynamic instability at concentrations that inhibited cell proliferation and induced mitotic arrest. It has also been reported that high consumption of brassicaceous vegetables is regularly associated with low cancer risk, with its extracts regulating the progression of cancer through anti-inflammatory effects, effects on signal transduction, epigenetic effects and modulation of the colonic microflora $^{(13)}$. At present, interest in a number of fruits high in polyphenols has been heightened because of their reported chemopreventive and chemotherapeutic potential ${ }^{(14)}$. Many fruits

\footnotetext{
Abbreviation: HR, hazard ratio.
}

* Corresponding author: Professor C.-X. Zhang, fax +86 208733 0446, email zhangcx3@mail.sysu.edu.cn 
such as pomegranate have been shown to exert anticancer activity, which is generally attributed to their high polyphenol content $^{(15)}$. Therefore, high consumption of fruits and vegetables might play an important role in breast cancer prognosis.

To clarify the effects of the consumption of fruits and vegetables on the prognosis of breast cancer survivors, several studies have investigated the association of fruit and vegetable intake with all-cause mortality or breast cancer-specific mortality or recurrence ${ }^{(10,16-26)}$. The majority of these studies have reported a non-significant inverse association of fruit and vegetable intake with all-cause mortality ${ }^{(17,22,24-26)}$, and two studies also found this same non-significant association with breast cancer recurrence ${ }^{(10,25)}$. Moreover, among survivors of early-stage breast cancer, adoption of a diet that was very high in vegetables, fruits and fibre and low in fat did not reduce additional breast cancer events or mortality during a 7.3-year follow-up period ${ }^{(27)}$. However, two studies reported an inverse association of fruit and vegetable intake with all-cause mortality $^{(16,21)}$, and one study reported that vegetable intake is inversely associated with breast cancer recurrence ${ }^{(18)}$. Hauner et $a l .{ }^{(28)}$ found that a low-fat diet rich in fruits, vegetables and fibre seems to be weakly associated with a better prognosis. To our knowledge, the findings on the effects of fruit and vegetable intake on breast cancer prognosis are not conclusive. Therefore, a meta-analysis of prospective cohorts is required to quantitatively evaluate the overall effect of fruit and vegetable intake on the prognosis of breast cancer.

\section{Methods \\ Literature search}

A comprehensive search of PubMed, Web of Science, OVID, ProQuest and Chinese databases from June 1955 to April 2016 was conducted. The structured search strategy included the following format of key terms: [(vegetable OR fruit OR diet OR nutrition)] AND [(breast cancer OR breast neoplasm OR breast carcinoma)] AND [(prognosis OR disease progression OR disease-free survival OR mortality OR survival rate OR survival analysis OR medical futility OR treatment outcome OR treatment failure OR causes of death OR fatal outcome OR recurrence)]. We also carried out manual searches of the reference lists of eligible articles and pertinent reviews. Only full-length, original articles were considered, and no attempt was made to include abstracts or unpublished results. No language restrictions were imposed. The titles and abstracts of all identified articles were screened by one investigator (C. P.) for eligibility. Two investigators (C. P. and W.-P. L.) independently reviewed the full texts of the remaining articles to identify eligible studies, with differences in opinion resolved by consensus.

\section{Study selection criteria}

Studies were eligible for the analysis if (i) the study design was a prospective cohort study; (ii) data related to the dietary consumption of fruits and vegetables were available; (iii) prognostic indicators were restricted to recurrence, all-cause mortality and breast cancer-specific mortality ${ }^{(29)}$; and (iv) the study provided OR, relative risk (RR) or hazard ratio (HR) and CI data associating fruit and vegetable intake with breast cancer prognosis ${ }^{(30-32)}$. When multiple publications covered the same population, only the most recently published report was included in the analysis. Case reports, reviews, articles without full text, animal studies and in vitro studies were also excluded.

\section{Data extraction and quality assessment}

Two reviewers (C. P. and W.-P. L.) independently reviewed and extracted the data. Any discrepancies between the two reviewers were resolved by discussion until a consensus was reached. The study characteristics were recorded using a standardised data collection form, which included the name of the first author, publication year, country or region, study design, number of cases, length of follow-up, dietary assessment, exposure or outcome, comparison, OR, RR or HR from the most fully adjusted model for the highest $v$. the lowest fruit and vegetable intakes and their corresponding 95\% CI; confounders were adjusted for in the multivariate analysis.

The quality of the included studies, including selection of the study population, the comparability of the study groups and the outcome or exposure assessment, was assessed on the basis of the Newcastle-Ottawa Quality assessment scale (NOS) for assessing the quality of non-randomised studies in metaanalysis $^{(33)}$. Studies were considered to be of high quality if they scored seven or higher of a possible nine points ${ }^{(34,35)}$.

\section{Statistical analysis}

Summary HR were calculated for recurrence and death (all-cause and breast cancer-specific mortality) using generic inverse variance. The generic inverse variance method in RevMan can be used for ratio measures such as $\mathrm{HR}$ or $\mathrm{RR}^{(36)}$. High $v$. low meta-analysis was performed with ratio data from the most fully adjusted model for the highest $v$. the lowest fruit and vegetable intakes. HR is used to represent ratio measures of effect, including RR. Adjusted HR estimates are summarised using a fixed or random effects model. The random effects model was used to consider both within-study and betweenstudy variations. Assessment of heterogeneity among the studies was performed by using $Q$ and $I^{2}$ statistics. $P$-values of $<0.05$ or $I^{2}$-values over $50 \%$ indicate substantial heterogeneity $^{(37)}$. If substantial heterogeneity exists, the random effects model is appropriate; otherwise, the fixed effects model is preferred ${ }^{(38)}$. Pre-specified stratified analyses were performed to assess the effects of the various study characteristics. Subgroup analyses were carried out by comparing summary HR in each stratum, including menopausal status, number of patients, countries, timing of dietary intake and the use of tamoxifen, on outcomes. Because of the small number of studies reporting on recurrence or breast cancer-specific mortality, the subgroup analyses were mainly carried out for the association between fruit and vegetable intake and all-cause mortality.

Population-based study cohorts were defined as highly representative ${ }^{(39)}$. In contrast, studies that selected groups of users, for example, nurses and volunteers, and studies with no 
description of the derivation were categorised as poorly representative ${ }^{(39)}$. To assess the stability of the findings, sensitivity analyses to rule out poorly representative results from a single study in the meta-analysis were carried out by excluding each study individually and using both fixed and random effects models to evaluate the robustness of the results. Tests for publication bias were performed by constructing and assessing funnel plots. A symmetric inverted funnel shape arises from a 'well-behaved' data set, in which publication bias is unlikely ${ }^{(40)}$.

\section{Results}

\section{Results of the literature search}

According to the search criteria, 4493 records were identified, of which $96 \%$ were excluded from the meta-analysis after reviewing the title and abstract. After reviewing the full text of the remaining 146 articles, twelve articles ${ }^{(10,16-26)}$ were included in the meta-analysis. The most common reasons for exclusion were lack of data on fruit and vegetable intake, breast cancer recurrence, all-cause mortality or breast cancer-specific mortality. A flow chart of the procedure used to select studies is shown in Fig. 1.

\section{Characteristics of studies}

The characteristics and quality scores of the included studies are summarised in Table 1. In total, twelve included studies involving 52962 women were identified, which included 29295 breast cancer patients from eleven studies ${ }^{(10,16-22,24-26)}$ and 23667 women from one study ${ }^{(23)}$. The studies had three outcomes: all-cause mortality in ten studies ${ }^{(10,16,17,19-22,24-26)}$, breast cancerspecific mortality in four studies ${ }^{(17,19,23,26)}$ and recurrence in three studies ${ }^{(10,18,25)}$. The twelve studies included prospective cohort studies published between 1999 and 2013. In total, seven studies were conducted in the USA ${ }^{(17,18,20-22,24,25)}$, one in China ${ }^{(16)}$, one in Japan ${ }^{(23)}$, one in Germany ${ }^{(26)}$, one in Italy ${ }^{(19)}$ and one in both the USA and China ${ }^{(10)}$.

The number of participants in each study ranged from 149 to 23667 , and the median length of follow-up ranged from 3 to 18 years. Most of the individual studies adjusted for a wide range of potential confounders, including age, BMI, stage of disease, age at diagnosis, hormone-replacement therapy use, alcohol use and physical activity. Most studies used a validated FFQ to assess the consumption of fruits and vegetables; eleven studies were of high quality (NOS score $\geq 7$ ), with an average NOS score of $8 \cdot 0$.

\section{All-cause mortality}

As shown in Fig. 2(a), three included studies ${ }^{(19,20,22)}$ with four groups of patients investigated the association between the highest $v$. the lowest intake of fruits and vegetables combined and all-cause mortality among breast cancer patients. The summary HR (highest $v$. lowest) from the three studies for all-cause mortality was $1.01(95 \%$ CI $0.72,1.42)$ with moderate heterogeneity ( $P_{\text {for heterogeneity }}=0.09, I^{2}=54 \%$ ). No significant association was found between fruit and vegetable intake and all-cause mortality among the breast cancer patients. The funnel plot was symmetrical, and there was no evidence of publication bias.

In all, seven included studies ${ }^{(16,17,21,22,24-26)}$ with eight groups of patients were eligible for the meta-analysis of the association between highest $v$. lowest total vegetable intake and all-cause mortality among breast cancer patients. The summary HR (highest $v$. lowest) from the eight groups of breast cancer patients for all-cause mortality was $0.71(95 \%$ CI $0.43,1.17)$ with evidence of high heterogeneity $\left(P_{\text {for }}\right.$ heterogeneity $<0.00001$, $\left.I^{2}=91 \%\right)$. However, in the funnel plot, one study ${ }^{(16)}$ deviated far from the vertical axis, and an asymmetrically inverted funnel shape indicates potential publication bias. Therefore, as shown in Fig. 2(b), this study ${ }^{(16)}$ was excluded, and the summary HR (highest $v$. lowest) from the remaining six studies was 0.96 (95\% CI $0.83,1.12$ ) with no significant heterogeneity ( $P_{\text {for heterogeneity }}=0 \cdot 11, I^{2}=42 \%$ ). No significant association was found between total vegetable intake and all-cause mortality among the breast cancer patients. There was no evidence of small-study bias from the funnel plot.

As shown in Fig. 2(c), three included studies ${ }^{(10,17,22)}$ with four groups of patients investigated the association between highest $v$. lowest cruciferous vegetable intakes and all-cause mortality. The summary HR (highest $v$. lowest) for all-cause mortality was 0.99 (95\% CI $0.89,1.11)$ with no significant heterogeneity ( $P_{\text {for heterogeneity }}=0 \cdot 84, I^{2}=0 \%$ ). No significant association was found between cruciferous vegetable intake and all-cause mortality among the breast cancer patients. The funnel plot was symmetrical, and there was no evidence of publication bias.

As shown in Fig. 2(d), six included studies ${ }^{(16,17,21,22,25,26)}$ with seven groups of patients investigated the association between highest $v$. lowest fruit intake and all-cause mortality. The summary HR (highest $v$. lowest) from the six studies for all-cause mortality was $0.88(95 \%$ CI $0.74,1.05)$ with no heterogeneity detected ( $P_{\text {for heterogeneity }}=0 \cdot 11, I^{2}=43 \%$ ). Thus, no significant association was found between fruit intake and all-cause mortality. The funnel plot was symmetrical, and there was no evidence of publication bias.

\section{Breast cancer-specific mortality}

As shown in Fig. 3(a), three included studies ${ }^{(17,23,26)}$ investigated the association between highest $v$. lowest total vegetable intake and breast cancer-specific mortality. The summary HR (highest $v$. lowest) for breast cancer-specific mortality was 1.05 (95\% CI $0.77,1.43$ ), with no evidence of heterogeneity ( $P_{\text {for heterogeneity }}=0 \cdot 82, I^{2}=0 \%$ ). Thus, no significant association was found between total vegetable intake and breast cancerspecific mortality. The funnel plot was symmetrical, and there was no evidence of publication bias.

As presented in Fig. 3(b), three included studies ${ }^{(17,23,26)}$ investigated the association between highest $v$. lowest fruit intake and breast cancer-specific mortality. The summary HR (highest $v$. lowest) for breast cancer-specific mortality was 0.94 (95\% CI $0.69,1.26)$, with no evidence of heterogeneity ( $P_{\text {for heterogeneity }}=0.54, I^{2}=0 \%$ ). Thus, no significant association was found between fruit intake and breast cancer-specific 


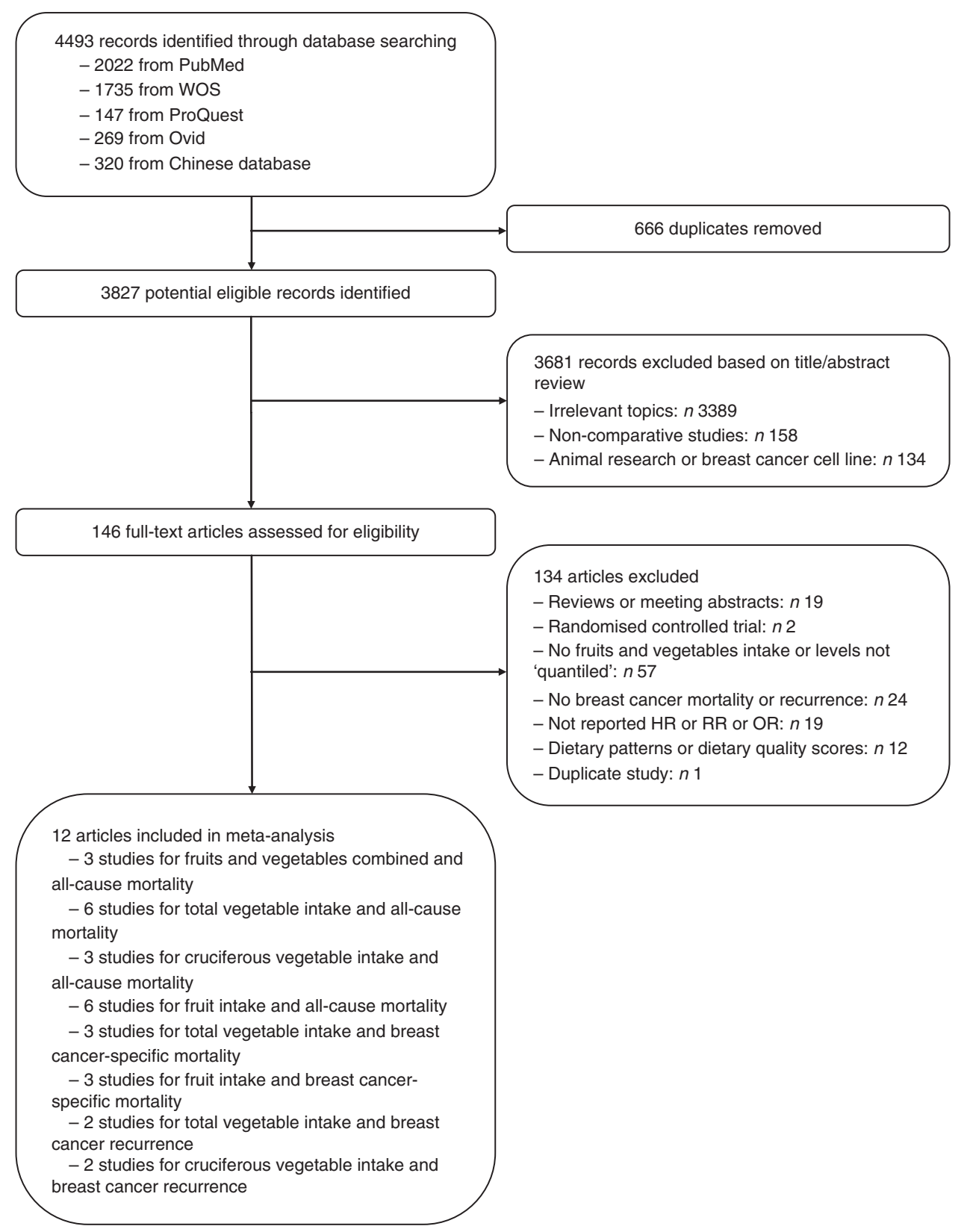

Fig. 1. Flow chart of the selection of studies included in the meta-analyses. WOS, Web of Science; HR, hazard ratio; RR, relative risk.

mortality. The funnel plot was symmetrical, and there was no evidence of publication bias.

\section{Breast cancer recurrence}

Two included studies ${ }^{(18,25)}$ investigated the association between highest $v$. lowest total vegetable intake and breast cancer recurrence. The summary HR (highest $v$. lowest) from these two studies for breast cancer recurrence was 0.89 (95\% CI 0.53, 1.50) with substantial heterogeneity shown $\left(P_{\text {for heterogeneity }}=\right.$ $0.003, I_{2}=89 \%$ ) in Fig. 4(a). There was no evidence of smallstudy bias from the funnel plot.

As presented in Fig. 4(b), two included studies ${ }^{(10,18)}$ investigated the association between highest $v$. lowest cruciferous vegetable intake and breast cancer recurrence. The summary HR (highest $v$. lowest) was 0.98 (95\% CI 0.76, 1.26) with moderate heterogeneity $\left(P_{\text {for heterogeneity }}=0.05, I^{2}=73 \%\right)$. The funnel plot was symmetrical, and there was no evidence of publication bias.

\section{Subgroup analyses}

Subgroup analyses of the associations of fruit and vegetable intake with all-cause mortality and breast cancer recurrence are summarised in Table 2.

Four included studies investigated the association between total vegetable intake ${ }^{(21,22,25,26)}$ or fruit intake ${ }^{(21,22,25,26)}$ and all-cause mortality stratified by menopausal status, respectively. 
Table 1. Characteristics of included studies and quality score

\begin{tabular}{|c|c|c|c|c|c|c|c|c|c|}
\hline $\begin{array}{l}\text { Author, } \\
\text { Publication year, } \\
\text { Country }\end{array}$ & Study name & Number of cases & $\begin{array}{l}\text { Follow-up period } \\
\text { (years) }\end{array}$ & $\begin{array}{l}\text { Dietary } \\
\text { assessment }\end{array}$ & Exposure & Contrast (highest $v$. lowest) & $\begin{array}{l}\mathrm{HR}(95 \% \mathrm{Cl}) \text { (highest } v \text {. } \\
\text { lowest) for mortality or } \\
\text { recurrence }\end{array}$ & Adjustments & NOS score \\
\hline $\begin{array}{c}\text { Beasley et al }{ }^{(17)} \\
2011 \text {, USA }\end{array}$ & CWLS & $\begin{array}{l}4441 \text { breast cancer } \\
\text { women }\end{array}$ & Mean 5.5 (SD 1.1) & $\begin{array}{l}\text { Validated 126- } \\
\text { item FFQ }\end{array}$ & $\begin{array}{l}\text { Vegetables; } \\
\text { Cruciferous } \\
\quad \text { vegetables; } \\
\text { Fruits }\end{array}$ & $\begin{array}{l}\text { Food group intake (quartiles) } \\
\text { (servings/d): } \\
\text { Vegetables: } Q 1=0.4 ; \\
\text { Q2=0.8; } \mathrm{Q} 3=1 \cdot 0 ; \mathrm{Q} 4=2.5 \\
\text { Cruciferous vegetables: } \\
\text { Q1=0.1; Q2 =0.2; Q3 =0.3; } \\
\text { Q4=0.7 } \\
\text { Fruits: Q1 } 1=0.1 ; \mathrm{Q} 2=0.4 ; \\
\text { Q3 }=1.0 ; \mathrm{Q} 4=2.5\end{array}$ & $\begin{array}{l}\text { All-cause survival: } \\
\text { Vegetables } 1.44(0.91,2.27) \text {; } \\
\text { Cruciferous vegetables } 1.02 \\
\text { (0.80, } 1.30) ; \\
\text { Fruits } 1.38(0.88,2.17) \\
\text { Breast cancer survival: } \\
\text { Vegetables } 0.96(0.38,2.45) ; \\
\text { Cruciferous vegetables } 0.95 \\
\text { (0.59, } 1.54) ; \\
\text { Fruits } 1.39(0.64,2.99)\end{array}$ & $\begin{array}{l}\text { Factors at diagnosis (age, state } \\
\text { of residence, menopausal } \\
\text { status, smoking status, } \\
\text { breast cancer stage, alcohol } \\
\text { intake, history of hormone- } \\
\text { replacement therapy), } \\
\text { interval between diagnosis } \\
\text { and diet assessment, and } \\
\text { factors at follow-up (energy } \\
\text { intake, breast cancer } \\
\text { treatment, BMI, and physical } \\
\text { activity) }\end{array}$ & 9 \\
\hline $\begin{array}{l}\text { Buck et al. }{ }^{(26)} \text {, } \\
\text { 2011, Germany }\end{array}$ & $\begin{array}{l}\text { Hamburg } \\
\text { RNK }\end{array}$ & $\begin{array}{l}2653 \text { post- } \\
\text { menopausal } \\
\text { breast cancer } \\
\text { women }\end{array}$ & Median 6.4 & $\begin{array}{l}\text { A self- } \\
\text { administered } \\
\text { validated } \\
\text { 176-items } \\
\text { FFQ }\end{array}$ & $\begin{array}{l}\text { Vegetables; } \\
\text { Fruits }\end{array}$ & $\begin{array}{l}\text { Vegetables }(n / \text { deaths })(\text { median } \\
\text { intake) } \\
\text { Tertile 1: } 875 / 118(79 \mathrm{~g} / \mathrm{d}) ; \\
\text { Tertile 2: } 902 / 100(118 \mathrm{~g} / \mathrm{d}) ; \\
\text { Tertile } 3: 876 / 103(183 \mathrm{~g} / \mathrm{d}) \\
\text { Fruits }(n / \text { deaths })(\text { median } \\
\text { intake) } \\
\text { Tertile 1: } 876 / 122(79 \mathrm{~g} / \mathrm{d}) ; \\
\text { Tertile 2: } 902 / 104(128 \mathrm{~g} / \mathrm{d}) \text {; } \\
\text { Tertile 3: } 876 / 95(259 \mathrm{~g} / \mathrm{d})\end{array}$ & $\begin{array}{l}\text { Overall mortality: } \\
\text { Vegetables } 1.09(0.80,1.48) \text {; } \\
\text { Fruits } 0.84(0.61,1.16) . \\
\text { Breast cancer mortality: } \\
\text { Vegetables } 1.01(0.70,1.46) \text {; } \\
\text { Fruits } 0.86(0.59,1.25)\end{array}$ & $\begin{array}{l}\text { Tumour size, nodal status, } \\
\text { metastasis, grade, ER/PR } \\
\text { status, breast cancer } \\
\text { detection type, diabetes, } \\
\text { menopausal hormone } \\
\text { therapy use at diagnosis, } \\
\text { study centre and energy } \\
\text { intake }\end{array}$ & 8 \\
\hline $\begin{array}{l}\text { Dal Maso et al. }{ }^{(19)} \text {, } \\
2008 \text {, Italy }\end{array}$ & PACE & $\begin{array}{l}1453 \text { invasive } \\
\text { breast cancer } \\
\text { women }\end{array}$ & $\begin{array}{l}\text { Median } 12 \cdot 6 \\
\quad(\text { maximum 16.8) }\end{array}$ & $\begin{array}{l}\text { Food frequency } \\
\text { consumption } \\
\text { section } \\
\text { (FFQ) }\end{array}$ & Vegetables and fruits & $\begin{array}{l}\text { Vegetables and fruits } \\
\text { (servings/d): } \\
\geq 6 ; 4 \text { to }<6 ;<4\end{array}$ & $\begin{array}{l}\text { All-cause mortality: } \\
\text { Vegetables and fruits } 1.27 \\
(1.00,1.61) . \\
\text { Breast cancer mortality: } \\
\text { Vegetables and fruits } 1.26 \\
(0.96,1.64)\end{array}$ & $\begin{array}{l}\text { Region of residence, age at } \\
\text { diagnosis, year of diagnosis, } \\
\text { TNM stage and ER/PR } \\
\text { status; Total energy intake, } \\
\text { using the residual method }\end{array}$ & 9 \\
\hline $\begin{array}{l}\text { Fink et al. }{ }^{(22)} \text {, } \\
2006 \text {, USA }\end{array}$ & LIBCSP & $\begin{array}{l}1235 \text { invasive } \\
\text { breast cancer } \\
\text { women }\end{array}$ & 3 & $\begin{array}{l}\text { Respondent- } \\
\text { completed, } \\
\text { modified } \\
\text { block FFQ }\end{array}$ & $\begin{array}{l}\text { Any fruits, fruit juices, } \\
\text { and vegetables; } \\
\text { Any vegetables; } \\
\text { Cruciferous vegetables; } \\
\text { Any fruits and fruit } \\
\text { juices; } \\
\text { Leafy vegetables; } \\
\text { Yellow vegetables; } \\
\text { Citrus fruits }\end{array}$ & $\begin{array}{l}\text { Any fruits, fruit juices and } \\
\text { vegetables: } 0-18 ; 19-25 ; \\
\text { 26-33; 34-45; 46+ } \\
\text { Any vegetables: 0-8; 9-13; } \\
\text { 14-17; 18-23; 24+ } \\
\text { Cruciferous vegetables: 0-1; 2; } \\
\text { 3; 4-5; 6+ } \\
\text { Any fruits and fruit juices: 0-6; } \\
\text { 7-12; 13-16; 17-23; 24+ } \\
\text { Leafy vegetables: 0-2; } 3 \text {; 4-5; } \\
\quad \text { 6-8; 9+ } \\
\text { Yellow vegetables: } 0-4 ; 5-7 ; \\
\text { 8-10; 11-15; 16+ } \\
\text { Citrus fruits: } 0-1 ; 2-4 ; 5-7 ; \\
\text { 8-11; } 12+\end{array}$ & $\begin{array}{l}\text { All-cause mortality: } \\
\text { Postmenopausal women } \\
\text { Any fruits, fruit juices, and } \\
\text { vegetables } 0.68(0.42,1.09) ; \\
\text { Any vegetables } 0.92(0.57, \\
1.48) ; \\
\text { Cruciferous vegetables } 1.07 \\
(0.67,1.72) ; \\
\text { Any fruits and fruit juices } 0.87 \\
(0.57,1.35) ; \\
\text { Leafy vegetables } 0.72 \\
\text { (0.41, } 1.24) ; \\
\text { Yellow vegetables } 0.90 \\
\text { (0.58, } 1.40) ; \\
\text { Citrus fruits } 0.90(0.56,1.44) \text {. } \\
\text { Premenopausal women } \\
\text { Any fruits, fruit juices and } \\
\text { vegetables } 1.38(0.65,2.91) ; \\
\text { Any vegetables } 1.40 \\
\text { (0.71, } 2.76) ; \\
\text { Cruciferous vegetables } 0.72 \\
\text { (0.34, } 1.54) ; \\
\text { Any fruits and fruit juices } 1.10 \\
(0.48,2.52) ; \\
\text { Leafy vegetables } 0.85 \\
(0.39,1.85) ; \\
\text { Yellow vegetables } 1.09 \\
\text { (0.52, } 2.28) ; \\
\text { Citrus fruits } 1.70 \text { ( } 0.75,3.89)\end{array}$ & Age and energy & 7 \\
\hline $\begin{array}{l}\text { Holmes et al. }{ }^{(24)} \text {, } \\
\text { 1999, USA }\end{array}$ & NHS & $\begin{array}{l}1982 \text { invasive } \\
\text { breast cancer } \\
\text { women }\end{array}$ & 18 & Validated FFQ & Vegetables & $\begin{array}{l}\text { Quantiles of vegetables intake } \\
\text { (servings): } \\
\text { Q1 } \leq 2 \cdot 12 \\
\text { Q2 }=2 \cdot 13-3 \cdot 12 \\
\text { Q3 }=3 \cdot 13-4 \cdot 20 \\
\text { Q4 }>4 \cdot 20\end{array}$ & $\begin{array}{l}\text { Multivariate RR for all-cause } \\
\text { mortality: } \\
\text { Vegetables } 0.81(0.59,1.11)\end{array}$ & $\begin{array}{l}\text { Age, diet interval, calendar } \\
\text { year of diagnosis, BMI, oral } \\
\text { contraceptive use, } \\
\text { menopausal status, smoking } \\
\text { status, age at first birth and } \\
\text { parity, postmenopausal } \\
\text { hormone use, number of } \\
\text { metastatic lymph nodes, } \\
\text { tumour size and energy } \\
\text { intake }\end{array}$ & 9 \\
\hline
\end{tabular}

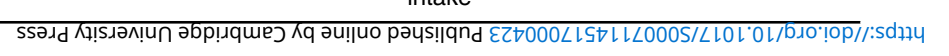




\begin{tabular}{|c|c|c|c|c|c|c|c|c|c|}
\hline $\begin{array}{l}\text { Author, } \\
\text { Publication year, } \\
\text { Country }\end{array}$ & Study name & Number of cases & $\begin{array}{l}\text { Follow-up period } \\
\text { (years) }\end{array}$ & $\begin{array}{l}\text { Dietary } \\
\text { assessment }\end{array}$ & Exposure & Contrast (highest $v$. lowest) & $\begin{array}{l}\mathrm{HR}(95 \% \mathrm{Cl}) \text { (highest } v \text {. } \\
\text { lowest) for mortality or } \\
\text { recurrence }\end{array}$ & Adjustments & NOS score \\
\hline $\begin{array}{l}\text { McEligot et al.(21), } \\
2006 \text {, USA }\end{array}$ & IRB & $\begin{array}{l}516 \text { post- } \\
\text { menopausal } \\
\text { breast cancer } \\
\text { women }\end{array}$ & $\begin{array}{l}\text { Mean } 80 \text { months } \\
\quad \text { (SD 18) }\end{array}$ & $\begin{array}{l}\text { 100-item NCl- } \\
\text { block FFQ }\end{array}$ & $\begin{array}{l}\text { Vegetables; } \\
\text { Fruits }\end{array}$ & $\begin{array}{l}\text { Vegetables (servings): } \\
\text { Tertile } 1=1 \cdot 0 ; \text { Tertile } 2=2 \text {; } \\
\text { Tertile } 3=3 \cdot 1 \\
\text { Fruits (servings): } \\
\text { Tertile } 1=1 \cdot 0 \text {; Tertile } 2=1 \cdot 1 \text {; } \\
\text { Tertile } 3=2\end{array}$ & $\begin{array}{l}\text { All-cause mortality: } \\
\text { Vegetables } 0.57(0.35,0.94) \\
\text { Fruits } 0.63(0.38,1.05)\end{array}$ & $\begin{array}{l}\text { Stage of disease, age at } \\
\text { diagnosis, BMI, parity, } \\
\text { hormone-replacement } \\
\text { therapy use, alcohol use, } \\
\text { multivitamin use and energy } \\
\text { intake }\end{array}$ & 8 \\
\hline $\begin{array}{l}\text { Nechuta et al. }{ }^{(10)}, \\
2013 \text {, USA, China }\end{array}$ & $\begin{array}{l}\text { ABCPP } \\
\text { SBCSS } \\
\text { LACE } \\
\text { WHEL } \\
\text { NHS }\end{array}$ & $\begin{array}{l}11390 \text { invasive } \\
\text { breast cancer } \\
\text { women }\end{array}$ & Median 9.0 & Validated FFQ & $\begin{array}{l}\text { Post-diagnosis } \\
\text { cruciferous } \\
\text { vegetables intake }\end{array}$ & $\begin{array}{l}\text { Post-diagnosis cruciferous } \\
\text { vegetable intake study- } \\
\text { specific quartiles (g/d) (Q1; } \\
\text { Q2; Q3; Q4): } \\
<10.7 ; 10.7-22.4 ; 22.4-43 \cdot 5 ; \\
43.5 \text { (LACE) } \\
<13.0 ; 13.0 \text { to <23.8; 23.8- } \\
\quad 44 \cdot 6 ; 44.6 \text { (WHEL) } \\
<15.4 ; 15.4-25.4 ; 25.4-43 \cdot 2 ; \\
\quad 43.2 \text { (NHS) } \\
<61.5 ; 61.5-93 ; 93-138 \cdot 0 ; \\
\text { 138.0 (SBCSS) }\end{array}$ & $\begin{array}{l}\text { Total mortality: } \\
\text { Cruciferous vegetables } 0.99 \\
\text { (0.86, } 1 \cdot 13) \text {. } \\
\text { Recurrence: } \\
\text { Cruciferous vegetables } 1.10 \\
(0.95,1.28)\end{array}$ & $\begin{array}{l}\text { Age at diagnosis, ER/PR } \\
\text { status, TNM stage, surgery, } \\
\text { chemotherapy, radiotherapy, } \\
\text { hormonal therapy, smoking } \\
\text { status, BMI, exercise, } \\
\text { menopausal status, race/ } \\
\text { ethnicity and education }\end{array}$ & 9 \\
\hline $\begin{array}{l}\text { Pierce et al. }{ }^{(20)} \text {, } \\
\text { 2007, USA }\end{array}$ & WHEL & $\begin{array}{l}1490 \text { early-stage } \\
\text { breast cancer } \\
\text { women }\end{array}$ & Average 6.7 & $\begin{array}{l}\text { 24-h dietary } \\
\text { recalls }\end{array}$ & $\begin{array}{l}\text { Vegetables-fruits/ } \\
\text { physical activity } \\
\text { (VF/PA) }\end{array}$ & $\begin{array}{l}\text { Low VF/low PA (mean, 3.1 VF } \\
\text { servings/d; } 221 \mathrm{MET}-\mathrm{min} / \\
\text { week) (reference); } \\
\text { Low VF/high PA (mean, 3.4 VF } \\
\text { servings/d; } 1478 \mathrm{MET}-\mathrm{min} / \\
\text { week); } \\
\text { High VF/low PA (mean, 7.2 VF } \\
\text { servings/d; } 224 \text { MET-min/ } \\
\text { week); } \\
\text { High VF/high PA category } \\
\text { (mean, 7.6 VF servings/d; } \\
\text { 1513 MET-min/week) }\end{array}$ & $\begin{array}{l}\text { Mortality: } \\
\text { Low VF and low PA } \\
\text { (reference); } \\
\text { High VF and low PA } 0.86 \\
(0.51,1.45)\end{array}$ & $\begin{array}{l}\text { Age, alcohol intake, tumour } \\
\text { hormone receptor status, } \\
\text { and time from diagnosis to } \\
\text { study entry }\end{array}$ & 7 \\
\hline $\begin{array}{l}\text { Sauvaget et all. }{ }^{(23)} \text {, } \\
2003 \text {, Japan }\end{array}$ & LSS & 23667 women & Median 16 & Validated FFQ & $\begin{array}{l}\text { Green-yellow } \\
\text { vegetables; Fruits }\end{array}$ & $\begin{array}{l}\text { Green-yellow vegetables and } \\
\text { fruit consumption were } \\
\text { classified into three } \\
\text { frequency categories: } \\
\text { C1 'once per week or less'; } \\
\text { C2 ' } 2-4 \text { times per week'; } \\
\text { C3 'daily or almost daily' }\end{array}$ & $\begin{array}{l}\text { RR for breast cancer mortality: } \\
\text { Green-yellow vegetables } \\
1.28(0.64,2.54) ; \\
\text { Fruits } 0.91(0.48,1.72) \text {. } \\
\text { RR for total cancer mortality: } \\
\text { Green-yellow vegetables } \\
0.92(0.84,1.01) ; \\
\text { Fruits } 0.88(0.80,0.96)\end{array}$ & $\begin{array}{l}\text { Sex, age, radiation dose, city, } \\
\text { BMI, smoking status, alcohol } \\
\text { intake and education level }\end{array}$ & 9 \\
\hline $\begin{array}{l}\text { Saxe et al. }{ }^{(25)} \text {, } \\
\text { 1999, USA }\end{array}$ & IRBSM & $\begin{array}{l}149 \text { women with } \\
\text { primary breast } \\
\text { cancer }\end{array}$ & Five or more & $\begin{array}{l}\text { A National } \\
\text { Cancer } \\
\text { Institute FFQ }\end{array}$ & $\begin{array}{l}\text { Green and yellow } \\
\text { vegetables; } \\
\text { Fruits }\end{array}$ & $\begin{array}{l}\text { Green and yellow vegetables } \\
\text { (per } 3 \text { servings/week) } \\
\text { Fruits (per } 14 \text { servings/week) }\end{array}$ & $\begin{array}{l}\text { Death }(n 26): \\
\text { Green-yellow vegetables } \\
0.97(0.70,1.35) ; \\
\text { Fruits } 1.06(0.69,1.63) . \\
\text { Recurrence }(n 28): \\
\text { Green-yellow vegetables } \\
1.17(0.90,1.52) ; \\
\text { Fruits } 0.96(0.63,1.45)\end{array}$ & Energy & 6 \\
\hline $\begin{array}{l}\text { Thomson et al. }{ }^{(18)} \text {, } \\
\text { 2011, USA }\end{array}$ & WHEL & $\begin{array}{l}3080 \text { breast cancer } \\
\text { women }\end{array}$ & 7.3 & $\begin{array}{l}\text { Pre-scheduled } \\
24-h \text { dietary } \\
\text { recalls }\end{array}$ & $\begin{array}{l}\text { Total vegetables; } \\
\text { Cruciferous vegetables }\end{array}$ & $\begin{array}{l}\text { Total vegetables: } \\
\text { Lowest tertile; middle tertile; } \\
\text { highest tertile } \\
\text { Cruciferous vegetables: } \\
\text { Lowest tertile; middle tertile; } \\
\text { highest tertile }\end{array}$ & $\begin{array}{l}\text { Recurrence } \\
\text { Vegetables } 0.69(0.55,0.87) \\
\text { Cruciferous vegetables } 0.85 \\
(0.69,1.06)\end{array}$ & $\begin{array}{l}\text { Time from diagnosis to study } \\
\text { entry, menopausal status, } \\
\text { clinical site, intervention } \\
\text { status, cancer stage, } \\
\text { oestrogen receptor status, } \\
\text { chemotherapy, BMl, physical } \\
\text { activity, and tamoxifen use in } \\
\text { the full model }\end{array}$ & 8 \\
\hline $\begin{array}{l}P^{P a n}{ }^{(16)} \text {, } \\
\text { 2003, China }\end{array}$ & $\begin{array}{l}\text { Survival follow- } \\
\text { up study }\end{array}$ & $\begin{array}{l}1406 \text { women with } \\
\text { primary breast } \\
\text { cancer }\end{array}$ & $\begin{array}{l}\text { Average 4.8; } \\
\text { Median 5.14 }\end{array}$ & FFQ & $\begin{array}{l}\text { Vegetables; } \\
\text { Fruits }\end{array}$ & $\begin{array}{l}\text { Vegetables: } 2.8 \% ; 24.6 \% ; \\
21.0 \% \\
\text { Fruits: } 5.3 \% ; 24.5 \% ; 31 \cdot 1 \% \\
\text { Significantly reduce: } \\
\quad \text { Reference; } \\
\text { No changes; } \\
\text { Slightly increase; } \\
\text { Significantly increase }\end{array}$ & $\begin{array}{l}\text { All-cause mortality: } \\
\text { Vegetables } 0.06(0.03,0.11) \text {, } \\
P<0.0001 \text {; } \\
\text { Fruits } 0.47(0.25,0.87) \\
P=0.001\end{array}$ & $\begin{array}{l}\text { Vegetables: intake of fruits and } \\
\text { intake of soya products } \\
\text { Fruits: intake of vegetables and } \\
\text { intake of soya products }\end{array}$ & 7 \\
\hline
\end{tabular}

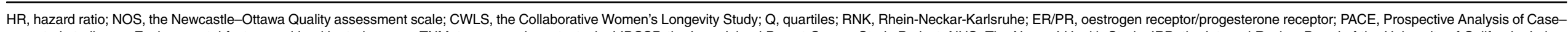

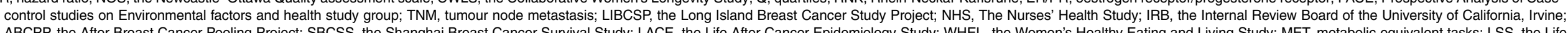

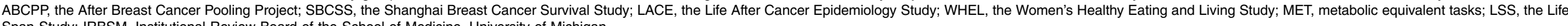
Span Study; IRBSM, Institutional Review Board of the School of Medicine, University of Michigan. 
(a)

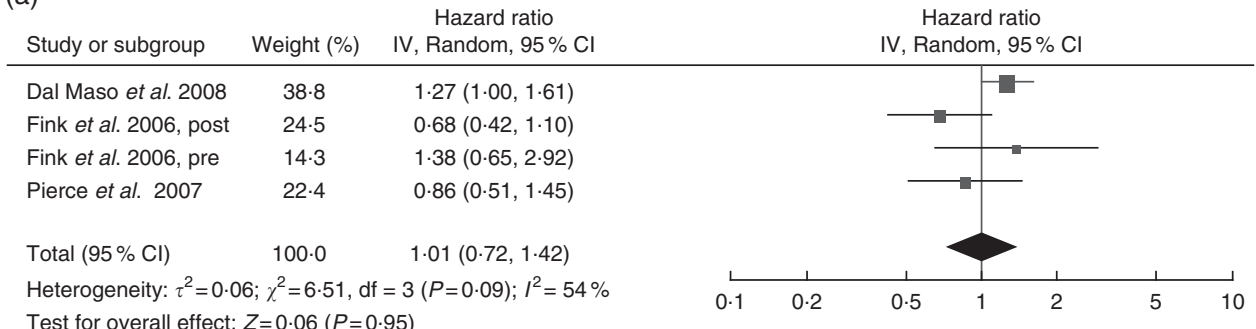

(b)

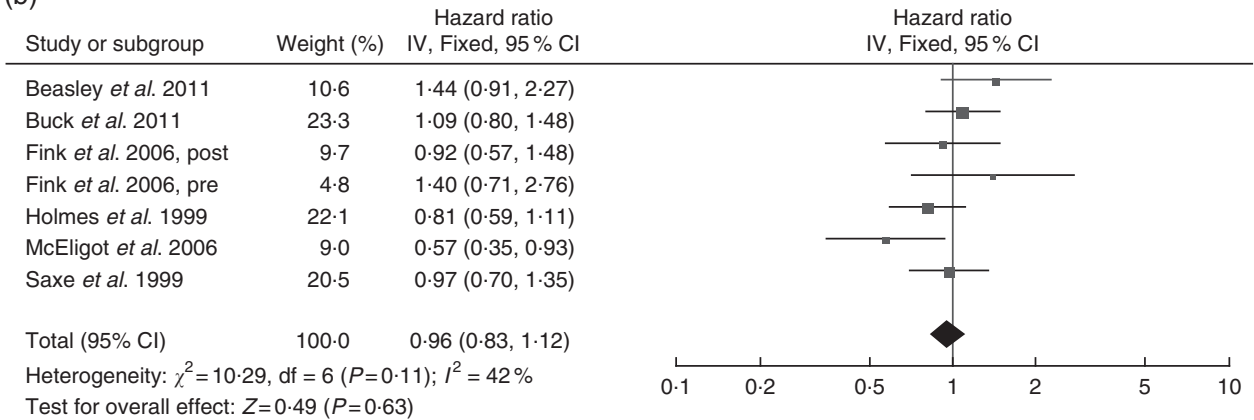

(c)

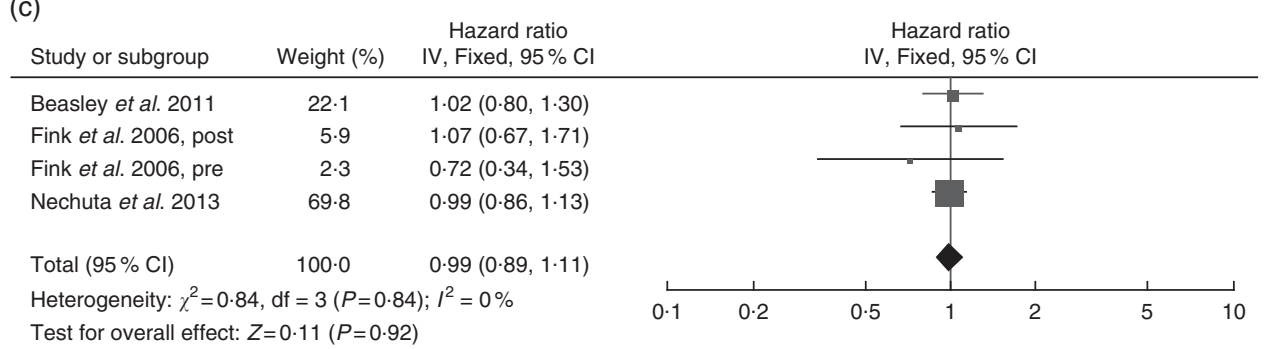

(d)

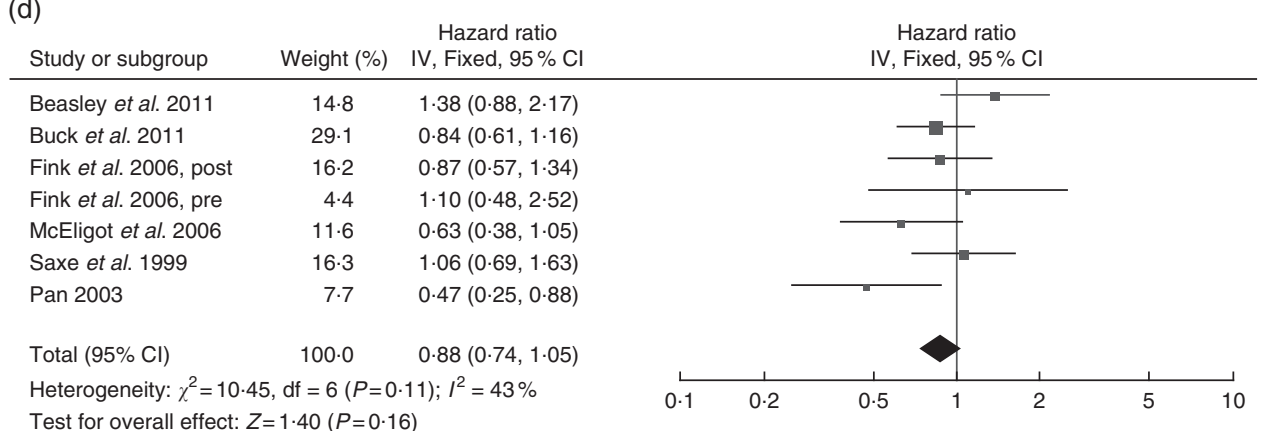

Fig. 2. Forest plots of observational studies investigating the association of all-cause mortality with (a) fruit and vegetable intake, (b) total vegetable intake, (c) cruciferous vegetable intake and (d) fruit intake. Highest $v$. lowest intake.

No significant association was found between total vegetable intake or fruit intake and all-cause mortality when stratified by menopausal status. The summary HR of total vegetable intake were 0.92 (95\% CI $0.76,1.12)$ for those who were postmenopausal and 1.18 (95\% CI $0.71,1.95)$ for those who were premenopausal. Those of fruit intake were 0.88 (95\% CI $0.72,1.08$ ) for postmenopausal patients and 0.90 (95\% CI 0.41 , 1.97) for premenopausal patients.

Six included studies with seven groups of patients were eligible for the meta-analysis of the association between total vegetable intake ${ }^{(17,21,22,24-26)}$ or fruit intake ${ }^{(16,17,21,22,25,26)}$ and all-cause mortality stratified by number of patients. Three studies included $4441^{(17)}, 1982^{(24)}$ and $2563^{(26)}$ breast cancer patients for total vegetable intake, and three studies included $1264^{(16)}$, $4441^{(17)}$ and $2563^{(26)}$ cases for fruit intake, whereas three other studies with four groups of patients included $516^{(21)}, 376^{(22)}$, $834^{(22)}$ and $149^{(25)}$ breast cancer patients for total vegetable or fruit intake. Stratified by number of patients, the summary HR of total vegetable intake were 1.05 (95\% CI $0.78,1.42)$ for studies with more than 1000 patients and 0.89 (95\% CI 0.66, 1.22) for those with less than 1000 patients. The summary HR of fruit intake were $0.85(95 \%$ CI $0.51,1.42)$ for those with more than 
(a)

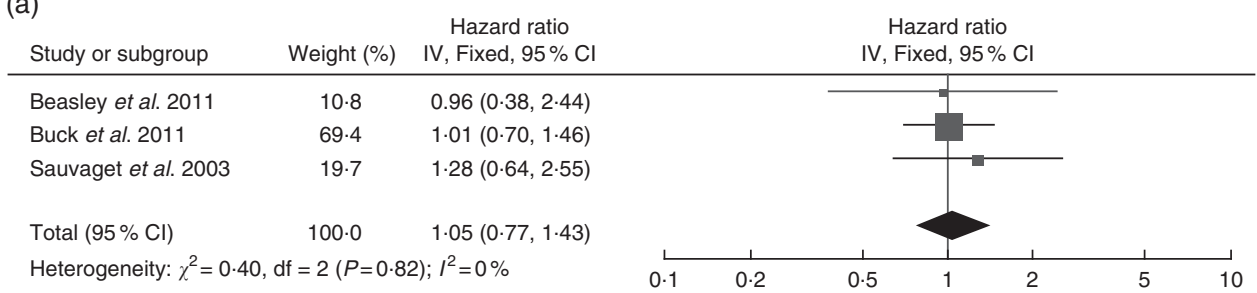

(b)

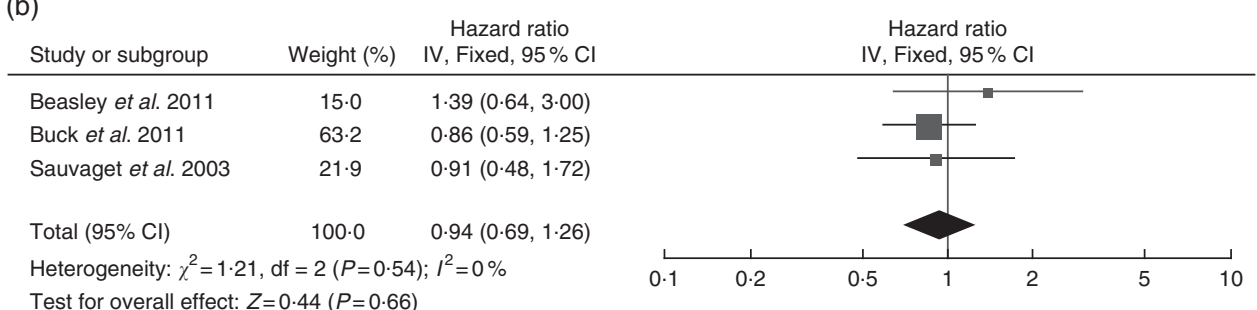

Fig. 3. Forest plots of observational studies investigating the association of breast cancer-specific mortality with (a) total vegetable intake and (b) fruit intake. Highest $v$. lowest intake.

(a)

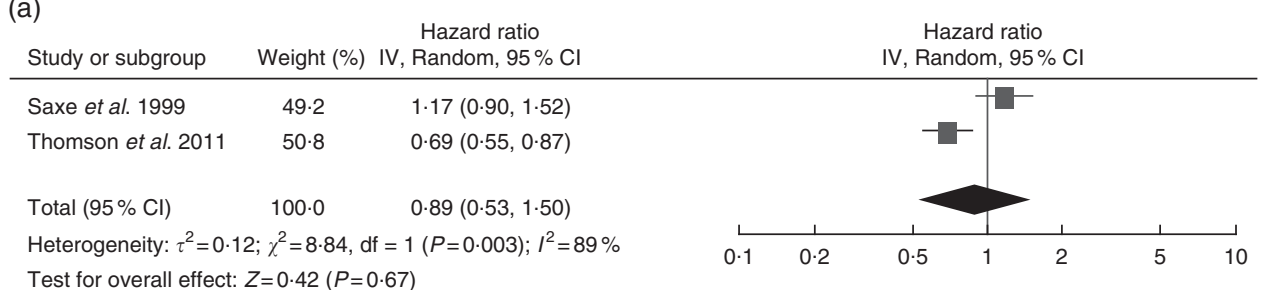

(b)

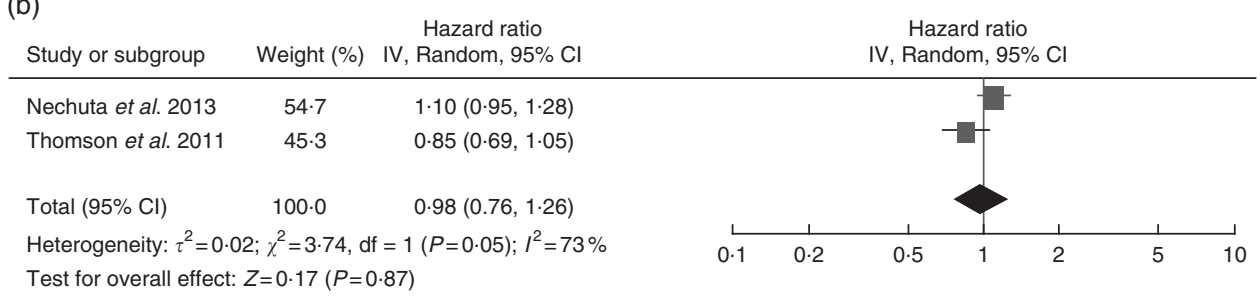

Fig. 4. Forest plots of observational studies investigating the association of breast cancer recurrence with (a) total vegetable intake and (b) cruciferous vegetable intake. Highest $v$. lowest intake.

1000 patients and $0 \cdot 88(95 \%$ CI $0 \cdot 68,1 \cdot 13)$ for those with less than 1000 patients.

Six included studies with seven groups of patients were eligible for the meta-analysis of the association between total vegetable

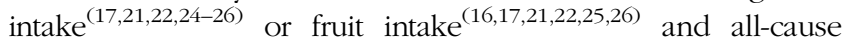
mortality stratified by timing of dietary intake information. Four studies $^{(21,22,25,26)}$ were based on information on diet collected before diagnosis or dietary habits before diagnosis and three studies collected after diagnosis ${ }^{(16,17,24)}$. The summary HR of total vegetable intake were 0.95 (95\% CI $0.75,1.20)$ for those whose information on diet was collected before diagnosis ${ }^{(21,22,25,26)}$ and 1.05 (95\% CI $0.60,1.85)$ for those collected after diagnosis ${ }^{(17,24)}$. The summary HR of fruit intake were 0.86 (95\% CI 0.71, 1.05) for those collected before diagnosis ${ }^{(21,22,25,26)}$ and 0.82 (95\% CI 0.29 , $2 \cdot 36)$ for those collected after diagnosis ${ }^{(16,17)}$.
Six included studies ${ }^{(16,17,21,22,25,26)}$ were eligible for the metaanalysis of the association between fruit intake and all-cause mortality stratified by countries. No significant association was found between fruit intake and all-cause mortality among breast cancer patients in the studies from the USA and the study from Germany, respectively. In the study data from the USA, the summary HR for all-cause mortality was 0.98 (95\% CI $0 \cdot 79,1 \cdot 21$ ). Of the single study from Germany and that from China, only the HR from the study in China indicated statistical significance.

Two included studies ${ }^{(10,18)}$ investigated the association between cruciferous vegetable intake and breast cancer recurrence stratified by the use of tamoxifen. No significant association was found between cruciferous vegetable intake and breast cancer recurrence when stratified by tamoxifen use. In the group of patients taking tamoxifen, the summary HR was 
Table 2. Summary of subgroup analyses of the effects of fruit and vegetable intake on all-cause mortality and breast cancer recurrence (Hazard ratios (HR) and $95 \%$ confidence intervals)

\begin{tabular}{|c|c|c|c|c|c|c|c|}
\hline \multirow[b]{2}{*}{ Outcomes } & \multirow[b]{2}{*}{ Groups } & \multirow[b]{2}{*}{ No. of studies } & \multicolumn{2}{|c|}{ Summary } & \multicolumn{2}{|c|}{ Heterogeneity } & \multirow[b]{2}{*}{$P$ for difference } \\
\hline & & & $\mathrm{HR}$ & $95 \% \mathrm{Cl}$ & $P(\%)$ & $P$ & \\
\hline \multicolumn{8}{|l|}{ All-cause mortality } \\
\hline \multirow[t]{9}{*}{ Total vegetable } & Menopausal states & 4 & & & & & 0.37 \\
\hline & Postmenopausal & 4 & 0.92 & $0.76,1.12$ & 38 & 0.19 & \\
\hline & Premenopausal & 2 & $1 \cdot 18$ & $0.71,1.95$ & 0 & 0.47 & \\
\hline & No. of patients $(n)$ & 6 & & & & & 0.46 \\
\hline & $>1000$ & 3 & 1.05 & $0.78,1.42$ & 55 & 0.11 & \\
\hline & $\leq 1000$ & 3 & 0.89 & $0.66,1.22$ & 41 & $0 \cdot 16$ & \\
\hline & Timing of dietary intake & 6 & & & & & 0.73 \\
\hline & Before diagnosis & 4 & 0.95 & $0.75,1.20$ & 35 & 0.19 & \\
\hline & After diagnosis & 2 & 1.05 & $0.60,1.85$ & 76 & 0.04 & \\
\hline \multirow[t]{13}{*}{ Fruit } & Menopausal status & 4 & & & & & 0.95 \\
\hline & Postmenopausal & 4 & 0.88 & $0.72,1.08$ & 24 & 0.27 & \\
\hline & Premenopausal & 2 & 0.90 & $0.41,1.97$ & 52 & 0.15 & \\
\hline & No. of patients $(n)$ & 6 & & & & & 0.90 \\
\hline & $>1000$ & 3 & 0.85 & $0.51,1.42$ & 74 & 0.02 & \\
\hline & $\leq 1000$ & 3 & 0.88 & $0.68,1.13$ & 0 & 0.45 & \\
\hline & Timing of dietary intake & 6 & & & & & 0.93 \\
\hline & Before diagnosis & 4 & 0.86 & $0.71,1.05$ & 0 & 0.61 & \\
\hline & After diagnosis & 2 & 0.82 & $0.29,2.36$ & 87 & 0.006 & \\
\hline & Countries & 6 & & & & & 0.09 \\
\hline & USA & 4 & 0.98 & $0.79,1.21$ & 29 & 0.23 & \\
\hline & Germany & 1 & 0.84 & $0.61,1.16$ & & & \\
\hline & China & 1 & 0.47 & $0.25,0.88$ & & & \\
\hline \multicolumn{8}{|l|}{$\mathrm{BC}$ recurrence } \\
\hline \multirow[t]{3}{*}{ Cruciferous vegetable } & Use of tamoxifen & 2 & & & & & 0.24 \\
\hline & Yes & 2 & $1 \cdot 12$ & $0.88,1.43$ & 0 & 0.70 & \\
\hline & No & 2 & 0.83 & $0.53,1.29$ & 82 & 0.02 & \\
\hline
\end{tabular}

HR, hazard ratio; BC, breast cancer.

${ }^{*} P$-values were obtained using test for subgroup differences to compare each group.

$1 \cdot 12(95 \%$ CI $0 \cdot 88,1 \cdot 43)$, whereas in the group of patients not taking tamoxifen the summary HR was 0.83 (95\% CI 0.53, 1.29).

\section{Sensitivity analyses}

Sensitivity analyses to examine the effects of outliers were performed by using both fixed and random effects models and excluding each study individually and re-calculating the summary HR. Most of the results showed that the summary HR of each meta-analysis remained statistically non-significant. Only the sensitivity analysis excluding one study ${ }^{(17)}$ yielded a different result, showing that the summary HR for the association of fruit intake with all-cause mortality was $0.82(95 \% \mathrm{CI}, 0.68,0.99)$ with no heterogeneity ( $P_{\text {for heterogeneity }}=0 \cdot 30, I^{2}=17 \%$ ), which may indicate the instability of the finding of the association between fruit intake and all-cause mortality (data not shown).

\section{Discussion}

The studies included in this meta-analysis examined four types of fruit and vegetable intakes including fruits and vegetables combined, total vegetable intake, cruciferous vegetable intake and fruit intake. The studies had three outcomes, including all-cause mortality, breast cancer-specific mortality and breast cancer recurrence. In this meta-analysis, no significant association was found between fruits and vegetables combined, total vegetable intake, cruciferous vegetable intake or fruit intake and all-cause mortality, breast cancer-specific mortality or breast cancer recurrence, respectively.

To our knowledge, this is the first meta-analysis to assess a possible association between fruit and vegetable intake and breast cancer prognosis. The ability of this meta-analysis to evaluate the effect of fruit and vegetable intake on breast cancer prognosis might be limited because the types of fruit and vegetable intake or the outcome indicators of breast cancer prognosis were investigated differently among the included studies. Some studies mainly investigated the association of total vegetable or fruit intake with all-cause mortality or breast cancer-specific mortality ${ }^{(17,21,23,24,26)}$, whereas others focused only on breast cancer recurrence $e^{(10,18,25)}$. Only one included study evaluated the association between fruits and vegetables combined $^{(19)}$ or cruciferous vegetable intake ${ }^{(17)}$ and breast cancer-specific mortality, respectively. Similarly, only one or no included study investigated the association between fruit intake ${ }^{(25)}$ or fruits and vegetables combined and breast cancer recurrence, respectively. Thus, the types of fruit and vegetable intake and the outcomes of breast cancer prognosis were inconsistent; therefore, this meta-analysis mainly evaluated the summary analysis on all-cause mortality.

The dose-response relationship of fruit and vegetable intake with all-cause mortality might be impossible to estimate in the present meta-analysis due to inconsistent assessment. Some studies used servings per day ${ }^{(17,19,20,21,24)}$ or quartiles with gram 
per day ${ }^{(10)}$, and others indicated a rough assessment criteria $^{(16,18,23)}$. Furthermore, some studies assessed the intake with quartiles ${ }^{(10,17,24)}$ and some with tertiles ${ }^{(21,26)}$. Most of the included studies indicated the HR of intake with highest $v$. lowest, but one study reported the HR with the highest quartile as the reference ${ }^{(19)}$. Although these factors constrained the ability to find a dose-response relationship and might slightly have influenced the summary HR investigated in this metaanalysis, sensitivity analyses were carried out. Most of them confirmed the robustness of the findings. Only the sensitivity analysis excluding one study ${ }^{(17)}$ investigating the relationship between fruit intake and all-cause mortality yielded different results, which showed that the excluded one ${ }^{(17)}$ might be poorly representative. However, no heterogeneity was detected on the summary HR for the association of fruit intake with all-cause mortality, which indicated that there is no difference among the included studies. Thus, this study ${ }^{(17)}$ was included for the meta-analysis.

The findings of this meta-analysis reported a non-significant association between fruit and vegetable intake and breast cancer prognosis, probably because the consumption of fruits and vegetables may not be a strong dietary determinant of breast cancer mortality but has an indirect effect on breast cancer prognosis ${ }^{(41,42)}$. Many related articles regarding the association of fruit and vegetable intake with breast cancer prognosis mainly focus on the association between fruit and vegetable intake and weight change among breast cancer patients ${ }^{(43-47)}$. Thomson et al. ${ }^{(48)}$ indicated minimal short-term changes in body weight in response to a high-vegetable, low-fat diet in breast cancer survivors. However, several studies have implicated obesity as being positively associated with breast cancer mortality ${ }^{(49-53)}$. Stokes et $a l^{(54)}$ indicated that an unhealthy body weight is an important modifiable risk factor for the recurrence of breast cancer. Hauner \& Hauner ${ }^{(55)}$ found that obesity is a potent risk factor for both cancer development and prognosis, increasing the risk for overall and breast cancerspecific mortality by approximately $30 \%$. On the whole, it is suggested that a healthy dietary pattern that increases fruit and vegetable intake and reduces dietary fat intake and is associated with modest weight loss may influence breast cancer prognosis. Thus, it is likely that the association of fruit and vegetable intake with breast cancer prognosis should be investigated with weight change among breast cancer patients.

Subgroup analyses stratified by menopausal states, number of patients and use of tamoxifen showed no different effects in each group. First, hormone-related factors such as late menopause or use of postmenopausal hormone therapy have been known to be associated with an increased risk of breast cancer $^{(56)}$. Bernstein \& Ross ${ }^{(57)}$ found that endogenous sex hormones play a role in the development of breast cancer. Further, of the natural forms of oestrogen in women, oestrone (E1) is predominately found in postmenopausal women, whereas oestradiol (E2) is the major form in women of reproductive age ${ }^{(58)}$. In addition, the clinical implications of oestrogen receptor and progesterone receptor are different between premenopausal and postmenopausal patients with breast cancer ${ }^{(59)}$. Some constituents mainly present in fruits and vegetables, such as phyto-oestrogens, may either have similar effects as oestrogen (agonistic) or block oestrogen's effects (antagonistic) $^{(60)}$. These compounds structurally resemble oestrogen and exert their effects primarily through binding to the oestrogen receptor ${ }^{(61)}$. However, not all included studies so far published had enough information on hormonal receptor breast cancer subtypes. Therefore, analysis stratified by hormonal receptor status cannot be conducted. The findings of the subgroup analyses stratified by menopausal status also did not show any statistical significance. More epidemiological studies are needed to further investigate the influence of sex hormone levels on which fruit and vegetable intake may impact in promoting breast cancer. Second, analysis stratified by the number of patients was conducted to determine potential sources of heterogeneity and to see whether this factor influences the results. No significant difference in the subgroup was found. Therefore, the number of patients did not seem to be relevant to explain variability between studies, which confirmed the robustness of the effects of total fruit or vegetable intake on all-cause mortality. Finally, tamoxifen is an important oestrogen receptor antagonist used successfully for the treatment and prevention of breast cancer ${ }^{(62)}$. One included study ${ }^{(18)}$ suggested that baseline vegetable intake may be associated with a reduction in the risk of breast cancer recurrence, particularly for those using tamoxifen, whereas another study ${ }^{(10)}$ found no significance in the presence or absence of tamoxifen. However, existing mechanistic evidence suggests a synergistic anti-carcinogenic action of combinational interventions with selected bioactive constituents in cruciferous vegetables and both tamoxifen and oestrogen receptor- $\alpha^{(63-65)}$. Thus, these findings encourage future investigations of the possible effects of fruits or vegetables on breast cancer prognosis.

In the subgroup analysis stratified by countries, the HR in the single study from China indicated a significant association between fruit intake and all-cause mortality. However, no significant association was indicated by the summary HR in the studies from the USA or in the study from Germany. Although breast cancer mortality rates have steadily increased in recent years, one study also found that east Asian countries have a lower rate, which may be associated with their low-fat diet and higher intake of fruits and vegetables when compared with their Western counterparts ${ }^{(3)}$. However, epidemiological studies evaluating the association of fruit and vegetable intake with breast cancer prognosis in Asia were limited in that only two included studies were from Asian countries ${ }^{(16,23)}$ in this meta-analysis. Thus, more epidemiological studies examining regional differences in diet are expected to explore the association between fruit intake and all-cause mortality among breast cancer patients, especially in Asian countries.

No significant association was found between total vegetable or total fruit intake and all-cause mortality when stratified by timing of collection of dietary intake information. Some researchers suggested that antioxidant supplements provided during treatment could repair cellular oxidative damage to cancer cells caused by treatments such as radiation therapy and chemotherapy ${ }^{(66,67)}$, which probably explain why fruit and vegetable intake following diagnosis may not be beneficial to breast cancer patients. However, Velentzis et al. ${ }^{(68)}$ reported that patients were likely to make significant changes to their diet 
and adopt healthier food choices after diagnosis of breast cancer, potentially decreasing their risk of breast cancer recurrence and reducing the risk of developing other co-morbidities including diabetes and heart disease, which probably account for all-cause mortality. Izano et al. ${ }^{(69)}$ indicated that healthy dietary choices after breast cancer diagnosis did not change the risk of breast cancer death and recurrence but were associated with a reduced risk of non-breast cancer mortality. Studies included in the present meta-analysis also indicated that high post-diagnostic fruit and vegetable intake decreased the risk of mortality following breast cancer ${ }^{(24,25)}$. Moreover, it is possible that pre-diagnosis diets may have reflected taste or appetite changes resulting from breast cancer ${ }^{(25)}$. Some studies indicated that pre-diagnosis diet associated with risk of breast cancer may be related to progression following the diagnosis of breast cancer and among the determinants of tissue characteristics that influence prognosis ${ }^{(70,71)}$. Dal et al. ${ }^{(19)}$ found that women eating $<4$ servings of fruits and vegetables/d before diagnosis showed higher risk for all-cause and breast cancer mortality compared with those who consumed 6 or more servings/d. Therefore, to pay more attention to post-diagnosis dietary changes and pre-morbid dietary habits in relation to breast cancer prognosis, more studies are needed to evaluate fruit and vegetable intake before and after diagnosis and its effects on breast cancer prognosis.

Although this meta-analysis provided no evidence that a higher fruit and vegetable intake decreased the risk of all-cause mortality among breast cancer patients, several studies indicated that dietary intakes of nutrients common in fruits and vegetables seem to be associated with a better prognosis among breast cancer patients. Fruits and vegetables are common sources of many candidate protective substances, including ascorbic acid and carotenoids ${ }^{(32)}$. Harris et al. ${ }^{(72)}$ suggested that post-diagnosis dietary vitamin $\mathrm{C}$ intake was statistically significantly associated with a reduced risk of total mortality and breast cancer-specific mortality. Plasma total carotenoid concentration was reported to be inversely associated with breast cancer recurrence in a cohort of 1551 women who had been diagnosed with early-stage breast cancer ${ }^{(73)}$. Dietary fibre was found to be inversely associated with overall mortality among breast cancer patients ${ }^{(21)}$. Moreover, some phytochemicals such as monoterpenes, resveratrol and lignans present in fruits and vegetables are also protective ${ }^{(74)}$. Some in vitro studies and animal models have also shown that nutrients from fruits and vegetables may inhibit the metastasis of breast cancer cells. Kim et al. ${ }^{(75)}$ found that a non-toxic, smallmolecule constituent of edible cruciferous vegetables (benzyl isothiocyanate) inhibits mammary cancer development in mouse mammary tumour virus-neu transgenic mice by causing epithelial tumour cell apoptosis. Koh et al. ${ }^{(76)}$ reported the significant inhibitory effect of lycopene on the invasive and migratory phenotypes of two highly aggressive breast cancer cell lines. $\mathrm{Xu}$ et $a l .{ }^{(77)}$ evaluated the effects of cyanidin-3glucoside $(\mathrm{C} 3 \mathrm{G})$, an anthocyanin present in many fruits and vegetables, on ethanol-induced breast cancer cell migration/ invasion and found that $\mathrm{C} 3 \mathrm{G}$ blocks ethanol-induced activation of the ErbB2/cSrc/FAK pathway, which is necessary for cell migration and invasion.
There are some potential limitations to this meta-analysis. First, only twelve articles ${ }^{(10,16-26)}$ with inconsistency in the types of fruit and vegetable intake and the prognostic indicators were included. Thus, the number of included articles might be too small to yield reliable summary results of breast cancer-specific mortality or breast cancer recurrence. Second, this metaanalysis did not estimate the dose-response relationship of fruit and vegetable intake with breast cancer prognosis and subgroup analysis stratified by other factors, such as pre- and post-diagnosis diet, because of lack of data. Finally, two funnel plots for the subgroup analyses stratified by menopausal state showed some asymmetry, indicating the risk of publication bias. However, the present meta-analysis also has several strengths. As all the included studies were prospective cohorts, we have effectively avoided recall and selection bias. Our clear delineation of the research questions and selection criteria, the comprehensive search strategy used and the objective assessment of the quality of the studies may have increased the validity of the findings. Moreover, with one exception, all the studies were high-quality cohorts. All but one of the sensitivity analyses yielded similar results, thus suggesting the stability of the findings. Most funnel plots were symmetrical, indicating that the results were unlikely to be due to publication bias.

In conclusion, no significant associations were found between fruit and vegetable intake (fruits and vegetables combined, total vegetable intake, cruciferous vegetable intake and fruit intake) and breast cancer prognosis (all-cause mortality, breast cancer-specific mortality and breast cancer recurrence). More studies are needed to determine whether the consumption of fruits and vegetables plays an important role in improving breast cancer prognosis. Additional studies on weight change, regional differences in diet, pre-diagnosis diet and dietary changes made after diagnosis are needed to confirm the effects of fruit and vegetable intake on breast cancer prognosis.

\section{Acknowledgements}

The authors gratefully acknowledge the student helpers for literature search in this study.

This study was supported by Science and Technology Program of Guangzhou, China (no. 201510010151), and the National Natural Science Foundation of China (no. 81102188). The funders had no role in the design, analysis or writing of this article.

The authors' responsibilities are as follows: C. P. conceived and designed the study, analysed the data, collected information and wrote the paper. W.-P. L. analysed the data and collected information. C.-X. Z. conceived and designed the study, analysed the data, collected information, supervised and contributed to writing of the paper.

The authors declare that there are no conflicts of interest.

\section{References}

1. Yue Y (2011) Research progress on risk factors of breast cancer. Oncology Progress 5, 520-523. 
2. McGuire A, Brown JA, Malone C, et al. (2015) Effects of age on the detection and management of breast cancer. Cancers $\mathbf{7}$, 908-929.

3. Torre LA, Bray F, Siegel RL, et al. (2015) Global cancer statistics, 2012. CA Cancer J Clin 65, 87-108.

4. Forman D \& Ferlay J (2014) The global and regional burden of cancer. In World Cancer Report 2014, pp. 16-53 [BW Stewart and CP Wild, editors]. Lyon, France: International Agency for Research on Cancer.

5. Brewster A \& Helzlsouer K (2001) Breast cancer epidemiology, prevention, and early detection. Curr Opin Oncol 13, $420-425$.

6. Reuben SC, Gopalan A, Petit DM, et al. (2012) Modulation of angiogenesis by dietary phytoconstituents in the prevention and intervention of breast cancer. Mol Nutr Food Res 56, 14-29.

7. Chatterjee M, Roy K, Janarthan M, et al. (2012) Biological activity of carotenoids: its implications in cancer risk and prevention. Curr Pharm Biotechnol 13, 180-190.

8. Liu J, Sun XR, Dong HW, et al. (2008) $\beta$-Ionone suppresses mammary carcinogenesis, proliferative activity and induces apoptosis in the mammary gland of the Sprague-Dawley rat. Int J Cancer 122, 2689-2698.

9. Liu JR, Dong HW, Sun XR, et al. (2010) Effects of beta-ionone on mammary carcinogenesis and antioxidant status in rats treated with DMBA. Nutr Cancer 62, 58-65.

10. Nechuta S, Caan BJ, Chen WY, et al. (2013) Postdiagnosis cruciferous vegetable consumption and breast cancer outcomes: a report from the After Breast Cancer Pooling Project. Cancer Epidemiol Biomarkers Prev 22, 1451-1456.

11. Fimognari C, Lenzi M \& Hrelia P (2008) Chemoprevention of cancer by isothiocyanates and anthocyanins: mechanisms of action and structure-activity relationship. Curr Med Chem $\mathbf{1 5}$, 440-447.

12. Azarenko O (2009) Dietary isothiocyanates suppress microtubule dynamic instability in association with mitotic arrest and inhibition of tumor cell proliferation, pp. 206. University of California, Santa Barbara, Ann Arbor.

13. Ferguson LR \& Schlothauer RC (2012) The potential role of nutritional genomics tools in validating high health foods for cancer control: broccoli as example. Mol Nutr Food Res 56, $126-146$

14. Turrini E, Ferruzzi L \& Fimognari C (2015) Potential effects of pomegranate polyphenols in cancer prevention and therapy. Oxid Med Cell Longev 2015, 938475.

15. Seeram NP, Adams LS, Henning SM, et al. (2005) In vitro antiproliferative, apoptotic and antioxidant activities of punicalagin, ellagic acid and a total pomegranate tannin extract are enhanced in combination with other polyphenols as found in pomegranate juice. $J$ Nutr Biochem 16, 360-367.

16. Pan ZT (2003) Study on survival and prognostic factors of female breast cancer in urban Shanghai. Masters thesis, Fudan University, Shanghai, China.

17. Beasley JM, Newcomb PA, Trentham-Dietz A, et al. (2011) Post-diagnosis dietary factors and survival after invasive breast cancer. Breast Cancer Res Treat 128, 229-236.

18. Thomson CA, Rock CL, Thompson PA, et al. (2011) Vegetable intake is associated with reduced breast cancer recurrence in tamoxifen users: a secondary analysis from the Women's Healthy Eating and Living Study. Breast Cancer Res Treat 125, 519-527.

19. Dal Maso L, Zucchetto A, Talamini R, et al. (2008) Effect of obesity and other lifestyle factors on mortality in women with breast cancer. Int J Cancer 123, 2188-2194.
20. Pierce JP, Stefanick ML, Flatt SW, et al. (2007) Greater survival after breast cancer in physically active women with high vegetable-fruit intake regardless of obesity. J Clin Oncol 25, 2345-2351.

21. McEligot AJ, Largent J, Ziogas A, et al. (2006) Dietary fat, fiber, vegetable, and micronutrients are associated with overall survival in postmenopausal women diagnosed with breast cancer. Nutr Cancer 55, 132-140.

22. Fink BN, Gaudet MM, Britton JA, et al. (2006) Fruits, vegetables, and micronutrient intake in relation to breast cancer survival. Breast Cancer Res Treat 98, 199-208.

23. Sauvaget C, Nagano J, Hayashi M, et al. (2003) Vegetables and fruit intake and cancer mortality in the Hiroshima/Nagasaki Life Span Study. Br J Cancer 88, 689-694.

24. Holmes MD, Stampfer MJ, Colditz GA, et al. (1999) Dietary factors and the survival of women with breast carcinoma. Cancer 86, 826-835.

25. Saxe GA, Rock CL, Wicha MS, et al. (1999) Diet and risk for breast cancer recurrence and survival. Breast Cancer Res Treat 53, 241-253.

26. Buck K, Zaineddin AK, Vrieling A, et al. (2011) Estimated enterolignans, lignan-rich foods, and fibre in relation to survival after postmenopausal breast cancer. Br J Cancer 105, 1151-1157.

27. Pierce JP, Natarajan L, Caan BJ, et al. (2007) Influence of a diet very high in vegetables, fruit, and fiber and low in fat on prognosis following treatment for breast cancer: the Women's Healthy Eating and Living (WHEL) randomized trial. JAMA 298, 289-298.

28. Hauner D, Janni W, Rack B, et al. (2011) The effect of overweight and nutrition on prognosis in breast cancer. Dtsch Arztebl Int 108, 795-801.

29. Li B, Lu Y, Wang L, et al. (2015) Folate intake and breast cancer prognosis. Eur J Cancer Prev 24, 113-121.

30. Aune D, Chan DS, Vieira AR, et al. (2012) Fruits, vegetables and breast cancer risk: a systematic review and meta-analysis of prospective studies. Breast Cancer Res Treat 134, 479-493.

31. Smith-Warner SA, Spiegelman D, Yaun SS, et al. (2001) Intake of fruits and vegetables and risk of breast cancer: a pooled analysis of cohort studies. JAMA 285, 769-776.

32. Gandini S, Merzenich H, Robertson C, et al. (2000) Metaanalysis of studies on breast cancer risk and diet: the role of fruit and vegetable consumption and the intake of associated micronutrients. Eur J Cancer 36, 636-646.

33. Zeng XT, Liu H, Chen X, et al. (2012) The fourth of meta analyses series: the quality assessment tool of observational studies. Chin J Evid Cardiovasc Med 4, 297-299.

34. Li Y, Yang H \& Cao J (2011) Association between alcohol consumption and cancers in the Chinese populationa systematic review and meta-analysis. PLOS ONE 6, 1451-1453.

35. Chak E, Rutherford GW \& Steinmaus C (2009) The role of breast-feeding in the prevention of Helicobacter pylori infection: a systematic review. Clin Infect Dis 48, 430-437.

36. The Australasian Cochrane Centre (2008) Part 5 Data and analyses: using generic inverse variance. In Review Manager 5.3 Tutorial (revised in June 2014), pp. 33-35. [staff at the Australasian Cochrane Centre and the Thai Cochrane Network and the Information Management System team (now IKMD, the Cochrane Informatics and Knowledge Management Department), editors]. Melbourne, Australia. http://community.cochrane.org/tools/ review-production-tools/revman-5/revman-5-download (accessed April 2016).

37. Higgins JP, Thompson SG, Deeks JJ, et al. (2003) Measuring inconsistency in meta-analyses. BMJ 327, 557-560.

38. DerSimonian R \& Laird N (1986) Meta-analysis in clinical trials. Control Clin Trials 7, 177-188. 
39. Wells GA, Shea B, O'Connell D, et al. (2016) The NewcastleOttawa Scale (NOS) for assessing the quality of nonrandomised studies in meta-analyses. http://www.ohri.ca/programs/ clinical_epidemiology/oxford.htm (accessed April 2016).

40. Sterne JA \& Egger M (2001) Funnel plots for detecting bias in meta-analysis: guidelines on choice of axis. J Clin Epidemiol 54, 1046-1055.

41. Hill MJ (1997) Nutrition and human cancer. Ann N Y Acad Sci 833, 68-78.

42. Verhoeven DT, Assen N, Goldbohm RA, et al. (1997) Vitamins C and $\mathrm{E}$, retinol, beta-carotene and dietary fibre in relation to breast cancer risk: a prospective cohort study. Br J Cancer $\mathbf{7 5}$, 149-155.

43. Erickson KD (2011) Diabetes and breast cancer: the women's healthy eating \& living study, pp. 96. University of California, Ann Arbor.

44. George SAM (2010) Energy balance, risk of breast cancer, and prognosis after breast cancer: investigating the complex roles of diet and physical activity, pp. 108. Yale University, Ann Arbor.

45. Thomson CA (1998) The effect of a high fruit and vegetable, low fat dietary intervention on immune function, DNA adduct formation, and body composition among breast cancer survivors, pp. 332. The University of Arizona, Ann Arbor.

46. Assaf AR, Beresford S, Risica PM, et al. (2016) Low-fat dietary pattern intervention and health-related quality of life: the women's health initiative randomized controlled dietary modification trial. J Acad Nutr Diet 116, 259-271.

47. Chlebowski R (2007) Lifestyle change including dietary fat reduction and breast cancer outcome. J Nutr 137S, 233S-235S.

48. Thomson CA, Rock CL, Giuliano AR, et al. (2005) Longitudinal changes in body weight and body composition among women previously treated for breast cancer consuming a highvegetable, fruit and fiber, low-fat diet. Eur J Nutr 44, 18-25.

49. Donegan WL, Hartz AJ \& Rimm AA (1978) The association of body weight with recurrent cancer of the breast. Cancer $\mathbf{4 1}$, 1590-1594

50. Reeves MM, Terranova CO, Eakin EG, et al. (2014) Weight loss intervention trials in women with breast cancer: a systematic review. Obes Rev 15, 749-768.

51. Rodriguez SFM, Aguilar MA \& Manuel-y-Keenoy B (2013) [Influence of body weight on the prognosis of breast cancer survivors; nutritional approach after diagnosis]. Nutr Hosp $\mathbf{2 8}$, 1829-1841.

52. Champ CE, Volek JS, Siglin J, et al. (2012) Weight gain, metabolic syndrome, and breast cancer recurrence: are dietary recommendations supported by the data? Int J Breast Cancer 2012, 506868.

53. McTiernan A, Irwin M \& Vongruenigen V (2010) Weight, physical activity, diet, and prognosis in breast and gynecologic cancers. J Clin Oncol 28, 4074-4080.

54. Stokes SA (2014) Dietary behaviors and nutrition intervention preferences of breast cancer survivors, pp. 73. Clemson University, Ann Arbor.

55. Hauner H \& Hauner D (2009) The impact of nutrition on the development and prognosis of breast cancer. Breast Care (Basel) 5, 377-381.

56. Key TJ, Verkasalo PK \& Banks E (2001) Epidemiology of breast cancer. Lancet Oncol 2, 133-140.

57. Bernstein L \& Ross RK (1993) Endogenous hormones and breast cancer risk. Epidemiol Rev 15, 48-65.

58. Liu MM, Huang Y \& Wang J (2012) Developing phytoestrogens for breast cancer prevention. Anticancer Agents Med Chem 12, 1306-1313.
59. Xiao JJ, Tao M, Duan WM, et al. (2009) Expressions of c-erbB-2, ER, PR and their prognostic correlation in premenopausal and postmenopausal patients with breast cancer. Cancer Res Prev Treat 36, 666-668.

60. Jung W, Yu O, Lau SM, et al. (2000) Identification and expression of isoflavone synthase, the key enzyme for biosynthesis of isoflavones in legumes. Nat Biotechnol 18, 208-212.

61. Turner JV, Agatonovic-Kustrin S \& Glass BD (2007) Molecular aspects of phytoestrogen selective binding at estrogen receptors. J Pharm Sci 96, 1879-1885.

62. Williams LD, Twaddle NC, Churchwell MI, et al. (2006) Quantification of tamoxifen and metabolites and soy isoflavones in human plasma using liquid chromatography with electrospray ionization tandem mass spectrometry. J Aoac Int 89, 1168-1173.

63. Malejka-Giganti D, Parkin DR, Bennett KK, et al. (2007) Suppression of mammary gland carcinogenesis by postinitiation treatment of rats with tamoxifen or indole3-carbinol or their combination. Eur J Cancer Prev 16, 130-141.

64. Wang TT, Milner MJ, Milner JA, et al. (2006) Estrogen receptor alpha as a target for indole-3-carbinol. J Nutr Biochem 17, 659-664.

65. Ramirez MC \& Singletary K (2009) Regulation of estrogen receptor alpha expression in human breast cancer cells by sulforaphane. J Nutr Biochem 20, 195-201.

66. Labriola D \& Livingston R (1999) Possible interactions between dietary antioxidants and chemotherapy. Oncology (Williston Park) 13, 1003-1008.

67. Lamson DW \& Brignall MS (1999) Antioxidants in cancer therapy; their actions and interactions with oncologic therapies. Altern Med Rev 4, 304-329.

68. Velentzis LS, Keshtgar MR, Woodside JV, et al. (2011) Significant changes in dietary intake and supplement use after breast cancer diagnosis in a UK multicentre study. Breast Cancer Res Treat 128, 473-482.

69. Izano MA, Fung TT, Chiuve SS, et al. (2013) Are diet quality scores after breast cancer diagnosis associated with improved breast cancer survival? Nutr Cancer 65, 820-826.

70. Rohan TE, Hiller JE \& McMichael AJ (1993) Dietary factors and survival from breast cancer. Nutr Cancer 20, $167-177$.

71. Russo J \& Russo IH (1994) Toward a physiological approach to breast cancer prevention. Cancer Epidemiol Biomarkers Prev 3, 353-364.

72. Harris HR, Orsini N \& Wolk A (2014) Vitamin C and survival among women with breast cancer: a meta-analysis. Eur $J$ Cancer 50, 1223-1231.

73. Rock CL, Flatt SW, Natarajan L, et al. (2005) Plasma carotenoids and recurrence-free survival in women with a history of breast cancer. J Clin Oncol 23, 6631-6638.

74. Tsubura A, Uehara N, Kiyozuka Y, et al. (2005) Dietary factors modifying breast cancer risk and relation to time of intake. J Mammary Gland Biol Neoplasia 10, 87-100.

75. Kim SH, Sehrawat A \& Singh SV (2013) Dietary chemopreventative benzyl isothiocyanate inhibits breast cancer stem cells in vitro and in vivo. Cancer Prev Res (Phila) 6 , 782-790.

76. Koh MS, Hwang JS \& Moon A (2010) Lycopene inhibits proliferation, invasion and migration of human breast cancer cells. Biomol Ther (Seoul) 18, 92-98.

77. Xu M, Bower KA, Wang S, et al. (2010) Cyanidin-3-glucoside inhibits ethanol-induced invasion of breast cancer cells overexpressing ErbB2. Mol Cancer 9, 1-14. 